\title{
Plasmonics: Localization and guiding of electromagnetic energy in metal/dielectric structures
}

\author{
Stefan A. Maier ${ }^{\text {a) }}$ and Harry A. Atwater \\ Thomas J. Watson Laboratories of Applied Physics, California Institute of Technology, Pasadena, \\ California 91125
}

(Received 17 September 2004; accepted 23 March 2005; published online 11 July 2005)

\begin{abstract}
We review the basic physics of surface-plasmon excitations occurring at metal/dielectric interfaces with special emphasis on the possibility of using such excitations for the localization of electromagnetic energy in one, two, and three dimensions, in a context of applications in sensing and waveguiding for functional photonic devices. Localized plasmon resonances occurring in metallic nanoparticles are discussed both for single particles and particle ensembles, focusing on the generation of confined light fields enabling enhancement of Raman-scattering and nonlinear processes. We then survey the basic properties of interface plasmons propagating along flat boundaries of thin metallic films, with applications for waveguiding along patterned films, stripes, and nanowires. Interactions between plasmonic structures and optically active media are also discussed. () 2005 American Institute of Physics. [DOI: 10.1063/1.1951057]
\end{abstract}

\section{TABLE OF CONTENTS}

I. INTRODUCTION...................

II. LOCALIZED PLASMON RESONANCES IN METAL NANOPARTICLES. ...............

A. Optical properties of single metal nanoparticles.....................

B. Interacting particle ensembles as a basis for applications of metal nanoparticles in optical devices...................

C. Local field enhancement around metal nanoparticle structures for sensing and nonlinear applications................

III. INTERFACE PLASMON POLARITONS AT METAL/DIELECTRIC BOUNDARIES. . . . . . . .

A. Surface-plasmon polaritons at metal

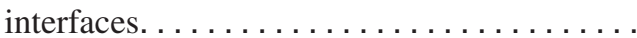

B. Metal stripes and nanowires:

Two-dimensional confinement. ..........

C. Apertures in a metallic screen............

D. Interactions with optically active media.....

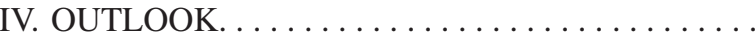

\section{INTRODUCTION}

The electromagnetic properties of metal/dielectric interfaces have attracted a vast amount of research effort ever since the work of $\mathrm{Mie}^{1}$ and Ritchie ${ }^{2}$ for small particles and flat interfaces, respectively. The ability of such structures to

\footnotetext{
${ }^{a}$ Present address: Department of Physics, University of Bath, Bath BA2 7AY, U.K.; electronic mail: s.maier@bath.ac.uk
}

sustain coherent electron oscillations known as surfaceplasmon polaritons (SPPs) leading to electromagnetic fields confined to the metallic surface has been intensively investigated ${ }^{3,4}$ both in light of the fundamental physics involved and for applications such as surface-enhanced spectroscopy and enhancement of nonlinear light generation. Af-

2 ter initial studies of the physics of these excitations, in the 1980s SPPs started to attract the attention of chemists, as the

2 electric-field enhancement around metal nanostructures was found to be crucial for surface-enhanced Raman spectroscopy.

More recently, the development of nanofabrication techniques such as electron-beam lithography, ion-beam milling, and self-assembly, together with modern nanocharacterization techniques such as dark-field and near-field optical microscopies and the emergence of quantitative electromagnetic simulation tools, has lead to a resurgence of interest in this field, ${ }^{5}$ partly due to potential applications for creating subwavelength optical devices enabling the miniaturization of optical components to size dimensions of their electronic counterparts, i.e, to the sub-100-nm-size regime. The unifying physical processes enabling light localization and guiding in such structures are the above-mentioned SPP excitations, and the name "plasmonics" for the subfield of modern optics studying such processes has been proposed. ${ }^{6}$

Due to the vast amount of research in this exploding field, ${ }^{5}$ we naturally had to select a rather small amount of topics for this review, leading to the omission of important applications of SPPs, for example, their use in integrated biological sensors based on multilayer structures, ${ }^{7}$ investigations from a more chemical viewpoint, ${ }^{8}$ as well as an indepth treatment of fabrication techniques. ${ }^{9}$ Here, we limit ourselves to a discussion of the fundamental physics of 


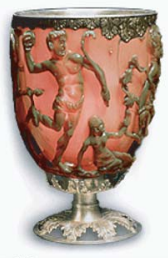

b)

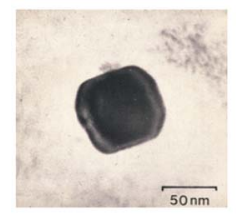

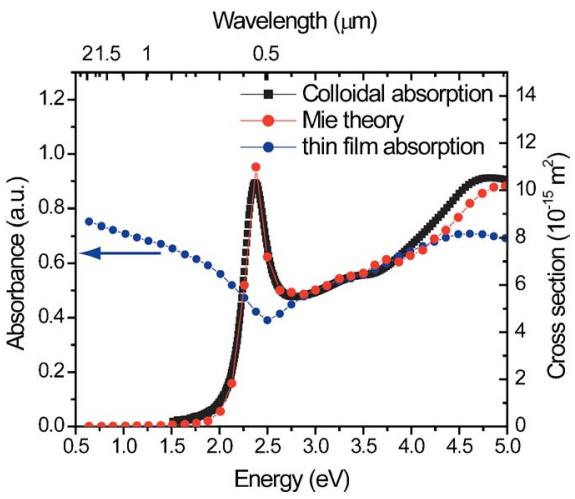

FIG. 1. (Color online) (a) The Lycurgus glass cup, demonstrating the bright red color of gold nanocrystals in transmitted light. (b) scanning electron microscopy (SEM) image of a typical nanocrystal embedded in the glass (courtesy of the British museum). (c) Calculated absorption spectrum of a thin gold film (blue dots) and of 30-nm Au nanoparticles in water (red dots) using classical electromagnetic theory. A measured absorption spectrum of an aqueous solution of 30-nm Au colloids (black dots) shows good agreement with the theory.

surface-plasmon excitations both for localized plasmons in metallic nanoparticles and for interface plasmons at metallodielectric film boundaries. A special focus has been put on the localization and guiding properties for electromagnetic radiation in light of applications of plasmon excitations for surface-enhanced spectroscopy such as sensing and higher harmonic generation and for the creation of a planar waveguide technology that can beat the diffraction limit.

\section{LOCALIZED PLASMON RESONANCES IN METAL NANOPARTICLES}

\section{A. Optical properties of single metal nanoparticles}

The strong interaction of microscopic metal particles of dimensions below $1 \mu \mathrm{m}$ with visible light has been employed for beautiful applications long before Gustav Mie's seminal 1908 paper Beiträge zur Optik trüber Medien, speziell kolloidaler Metallösungen (contributions to the optics of turbid media, particularly solution of colloidal metals). ${ }^{1}$ Historically, one prominent use of metal nanoparticles has been the staining of glass windows and ceramic pottery as seen in Fig. 1(a) by example of the Lycurgus cup (Byzantine empire, 4th century A. D.). The glass cup, on display in the British Museum, shows a striking red color when viewed in transmitted light, while appearing green in reflection. This peculiar behavior is due to small Au nanoparticles embedded in the glass [Fig. 1(b)], which show a strong optical absorption of light in the green part of the visible spectrum [Fig. 1(c)].

Indeed, the optical properties of metal nanoparticles, especially those of the noble metals $\mathrm{Au}, \mathrm{Ag}$, and $\mathrm{Cu}$, show striking differences relative to their bulk or thin-film optical responses. As an example, Fig. 1(c) shows the calculated absorption of a thin Au film (blue dots), as well as that of 30-nm Au spheres immersed in water (red dots), where the dispersion properties of $\mathrm{Au}$ have been modeled using measured dielectric data for bulk Au. ${ }^{10}$ For the nanoparticles, the optical-absorption spectrum has been obtained by directly solving Maxwell's equations for the scattering of electromagnetic waves by spherical objects as carried out by Mie, ${ }^{1}$ and retaining only the dipolar term, which is suitable for nanoparticles with a diameter $d \ll \lambda$, where $\lambda$ is the wavelength of light in the surrounding medium. As shown, this quasistatic approximation is in good agreement with measurements (black dots), which has been confirmed via a plethora of studies of the optical response of metallic nanoparticles with a diameter well below $\lambda$ in solid, liquid, and gaseous environments. ${ }^{4}$ Figure 1 (c) further demonstrates a striking difference between the optical response of the thin film and the nanoparticles. Whereas the film absorbs light throughout the near-infrared and visible regions due to freeelectron absorption, for the nanoparticles this process is strongly quenched for energies lower than $2 \mathrm{eV}$ (corresponding to wavelengths larger than $620 \mathrm{~nm}$ ). Indeed, all the freeelectron oscillator strength for absorption is pulled into a dipolar absorption peak around $2.25 \mathrm{eV}$, the dipolar surfaceplasmon particle resonance. This modified optical response leads to the bright colors of noble-metal nanoparticles, a nice discussion of which can be found in Ref. 11. For higher energies above the dipole resonance, the optical absorption of particles and films is similar, due to the dominance of $d-s p$ electronic interband transitions, which are prominent for $\mathrm{Au}$ and $\mathrm{Cu}$ in the vicinity of the dipole plasmon resonance, but less so for Ag.

The resonant electromagnetic behavior of noble-metal nanoparticles is due to the confinement of the conduction electrons to the small particle volume. For particles with a diameter $d \ll \lambda$, the conduction electrons inside the particle move all in phase upon plane-wave excitation with radiation of wavelength $\lambda$, leading to the buildup of polarization charges on the particle surface. These charges act as an effective restoring force, allowing for a resonance to occur at a specific frequency - the particle dipole plasmon frequency-, where the response of the electrons shows a $\pi / 2$ phase lag with respect to the driving field. Thus, a resonantly enhanced field builds up inside the particle, which in the small particle limit is homogeneous throughout its volume, producing a dipolar field outside the particle. This leads to enhanced absorption and scattering cross sections for electromagnetic waves, as well as to a strongly enhanced near field in the immediate vicinity of the particle surface. It is this resonantly enhanced near field from which most of the promising applications of metal nanoparticles stem. For larger particles, the spectral response is modified due to retardation effects and the excitation of higher-order (quadrupole and higher) modes, the spectral signature of which can be calculated by retaining higher orders of the Mie theory scattering coefficients. ${ }^{1}$

In general, the spectral position, damping, and strength of the dipole as well as of the higher-order plasmon resonances of single metal nanoparticles depend on the particle material, size, geometry, and the dielectric function of the surrounding host. ${ }^{4}$ For theoretical considerations, the large variety of naturally occurring or synthesized shapes of nanoparticles is often approximated via spheres or spheroids, for which analytically exact solvable solutions exist to all orders. ${ }^{1,4,12,13}$ The analysis is further facilitated for particles 


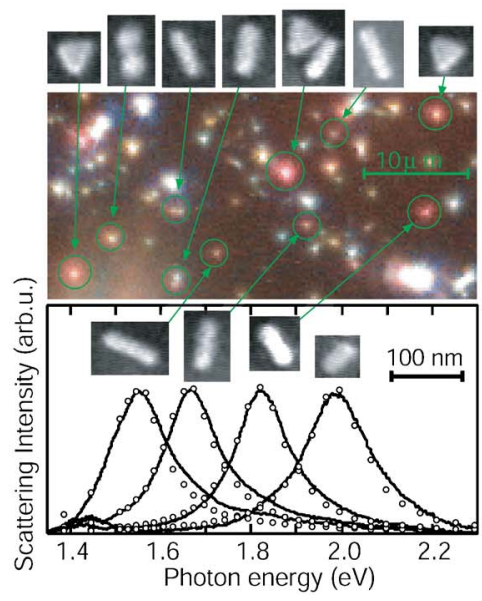

b)

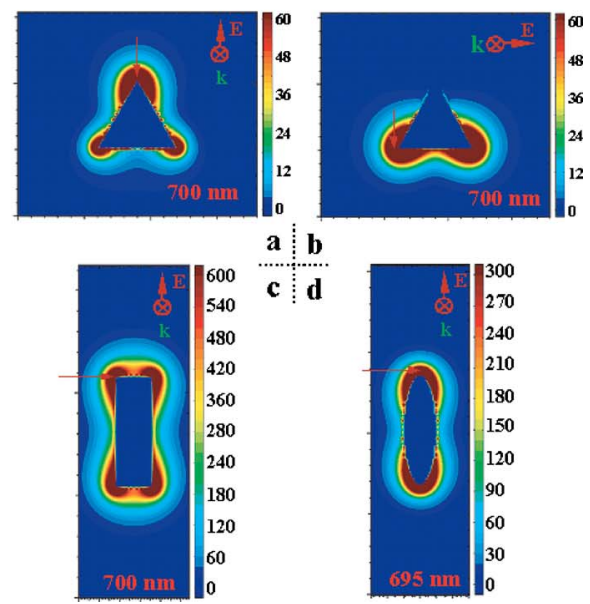

FIG. 2. (Color online) (a) Dark-field microcopy image (top) and lightscattering spectra (bottom) of $\mathrm{Au}$ nanocrystals of different shapes (adapted from Ref. 17). The measured spectra (black curves) show good agreement with predictions from a simple analytical extension of quasistatic Mie theory (open circles). (b) Electric near-field profile of the lowest-order modes of $\mathrm{Ag}$ nanoprisms calculated using the discrete dipole approximation formalism (adapted from Ref. 54). much smaller than the wavelength of light, where only the lowest (dipolar) order of the modal expansion of the scattered fields has to be retained. In this case, a quasistatic approach serves well to describe the spectral position, width, and strength of the dipolar plasmon resonance, as pointed out in the discussion of Fig. 1. For a spherical metal nanoparticle of radius $a \ll \lambda$ embedded in a nonabsorbing surrounding medium of dielectric constant $\varepsilon_{m}$, the quasistatic analysis gives the following expression for the particle polarizability $\alpha$ :

$$
\alpha=4 \pi a^{3} \frac{\varepsilon-\varepsilon_{m}}{\varepsilon+2 \varepsilon_{m}},
$$

with the complex $\varepsilon=\varepsilon(\omega)$ describing the dispersive dielectric response of the metal. The polarizability and thus the induced homogeneous polarization inside the particle are resonantly enhanced at the Fröhlich frequency where the denominator shows a minimum, limited by the imaginary part of $\varepsilon$ describing Ohmic heating losses within the particle. These losses are due to the creation of electron-hole pairs, the energy of which is subsequently coupled to the phonon bath. ${ }^{14}$ The spectral position of this resonance is seen to redshift with increasing dielectric constant of the surrounding host due to the buildup of polarization charges on the dielectric side of the interface, thus weakening the total restoring force. For ellipsoidal particles with principal axes $a, b$, and $c$, an analogous expression can be found in the quasistatic approximation via introducing geometrical depolarization factors $L_{i}$ along these axes, ${ }^{4,12}$ leading to

$$
\alpha=\frac{4}{3} \pi a b c \frac{\varepsilon-\varepsilon_{m}}{\varepsilon_{m}+L_{i}\left(\varepsilon-\varepsilon_{m}\right)}, \quad \sum L_{i}=1 .
$$

For spherical particles, $L_{1}=L_{2}=L_{3}=1 / 3$. For spheroidal particles $\left(L_{1}=L_{2}\right)$, the plasmon resonance thus splits into a strongly redshifted long-axis mode (polarization parallel to the long axis) and a slightly blueshifted short axis mode (polarization perpendicular to the long axis). ${ }^{12}$

For larger particles beyond the Rayleigh approximation, the dipolar resonance redshifts while at the same time suffering substantial broadening. The redshift is due to a reduction of the depolarization field due to retardation effects ${ }^{15}$ - the conduction electrons do not all move in phase anymore, leading to a reduced depolarization field at the particle center generated by the surrounding polarized matter. Additionally, radiative losses ${ }^{16}$ begin to significantly contribute to the plasmon damping, dominating the total damping of $\mathrm{Au}$ and $\mathrm{Ag}$ nanoparticles for particle sizes in excess of $100 \mathrm{~nm}$. The depolarization field and radiation damping effect can be seen as lowest-order corrections to the quasistatic theory, leading to additional real and imaginary parts of the denominator of the polarizability. A generalization of the quasistatic approach to particles of arbitrary shape has been suggested, with surprisingly good results ${ }^{17}$ [see Fig. 2(a)]. For particles with a diameter smaller than the free-electron scattering length, scattering processes at the particle surface are thought to begin to contribute to the total damping. ${ }^{4}$ These additional damping mechanisms for large and small particles lead to respective decreases in the total enhancement of the exciting field via a decrease of the plasmon dephasing time $T_{2}{ }^{4}$

Generally, numerical methods such as the $T$-matrix method, ${ }^{18}$ the discrete dipole approximation ${ }^{19}$ [Fig. 2(b)], or finite-difference time-domain simulations ${ }^{20}$ have to be used to calculate the resonance frequencies and mode profiles of more complex shapes. Such simulations have especially been employed to determine the local-field enhancement at the particle surface, in conjunction with discussions of enhancements of nonlinear processes and surface-enhanced Raman scattering (SERS) as discussed below.

Experimentally, sophisticated modern fabrication methods allow for the fabrication of metal nanoparticles and other nanostructures of a variety of shapes using both colloidal synthesis methods $^{21}$ and top-down nanofabrication techniques such as electron-beam lithography, ${ }^{22}$ and a wide variety of methods for the fabrication of metallic nanoparticles and ensembles thereof have recently been described in a different review article. ${ }^{9}$ The good control over the size and shape of the particles provided by these methods method allows one generally to observe homogeneously broadened line shapes of dipolar ${ }^{23}$ and multipolar ${ }^{24}$ plasmon modes in particle ensembles using conventional far-field spectroscopy. The direct examination of single particles has been demonstrated using both dark-field ${ }^{25}$ and near-field optical microscopies. ${ }^{26}$ The former method allows for a dramatic visualization of the spectral properties of single particles, as can be seen by the example in Fig. 2(a). 
a)

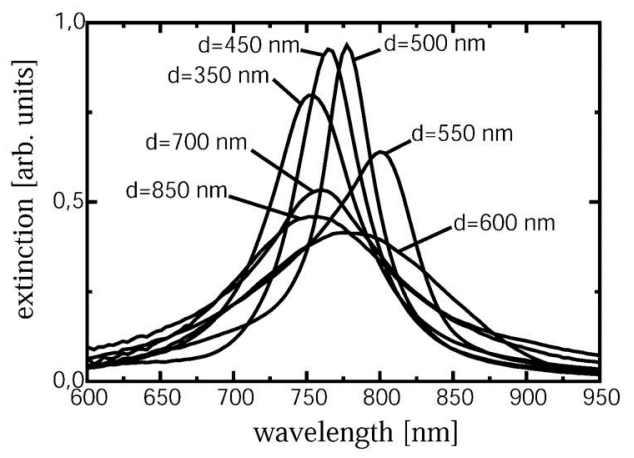

b)

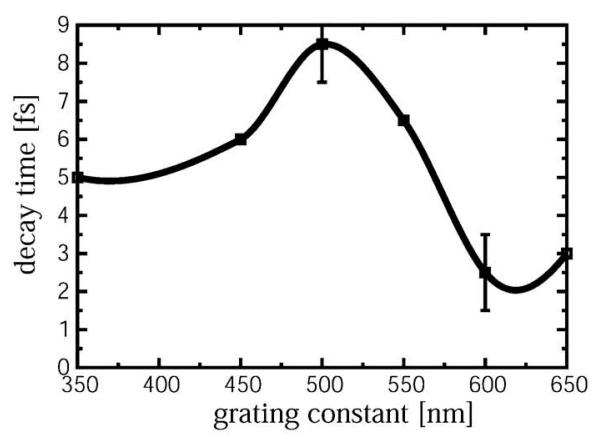

c)

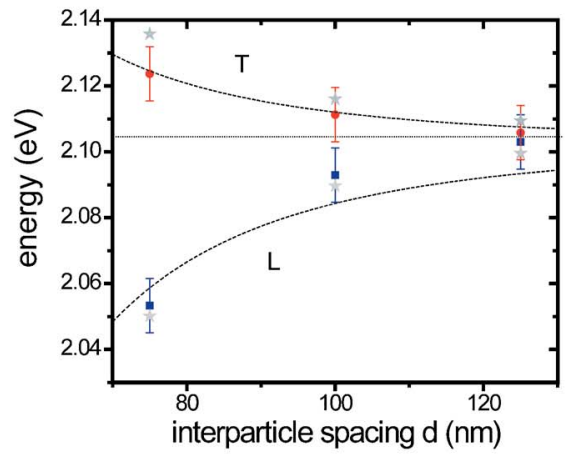

d)

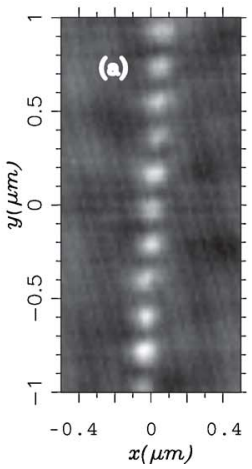

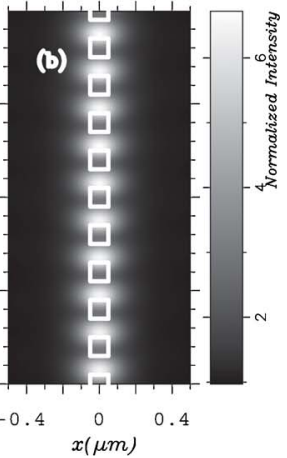

FIG. 3. (Color online) (a,b) Measured extinction spectrum (a) and plasmon decay time (b) for regular twodimensional (2D) square arrays of $\mathrm{Au}$ nanoparticles (adapted from Ref. 29, copyright by the American Physical Society). Both the spectral position and the decay time of the collective dipolar plasmon mode show a marked variation with grating constant due to far-field dipolar interactions. (c) Measured spectral position of the collective plasmon resonances of onedimensional arrays of closely spaced $\mathrm{Au}$ nanoparticles for longitudinal (L) and transverse polarizations (T). Also shown are results of a simple nearfield point-dipolar coupling model (solid lines) and finite-difference timedomain simulations (stars). (d) Optical near-field around such a chain obtained using collection mode near-field optical microscopy (left) and numerical simulations (right), adapted from Ref. 32 .

\section{B. Interacting particle ensembles as a basis for applications of metal nanoparticles in optical devices}

Advances in particle synthesis and fabrication techniques (for example, Refs. 22, 27, and 28) have recently allowed for studies of ordered arrays of noble-metal nanoparticles. In such arrays, each nanoparticle with a diameter much smaller than the wavelength $\lambda$ of the exciting light acts as an electric dipole. Thus, two types of electromagnetic interactions between the particles can be distinguished, depending on the spacing $d$ between adjacent nanoparticles. For particle spacings on the order of the exciting wavelength $\lambda$, far-field dipolar interactions with a $d^{-1}$ dependence dominate. Work on regular two-dimensional arrays of Au nanoparticles has indeed confirmed the existence of such interactions, and quantified their influence on both the spectral position of the collective dipolar extinction peak and the plasmon damping characteristics. ${ }^{29}$ Figures $3(\mathrm{a})$ and 3(b) show an example of the dependence of both extinction peak and plasmon decay time on the grating constant $d$ for a regular square array of 150-nm-diameter Au nanoparticles. Both the variation of the spectral position and width of the resonances can be explained by assuming far-field dipolar interactions - the ensemble acts effectively as a grating, leading to increased radiation damping of the collective resonances for grating constants where grating orders change from evanescent to radiative in character. ${ }^{29}$ Applications of such ordered arrays lie, for example, in the possibility of maximizing surface-enhanced Raman scattering of adsorbed molecules by careful spectral tuning of the plasmon resonance. $^{30}$

For particle spacings much smaller than the wavelength of light, near-field dipolar interactions between adjacent par- ticles with a distance dependence of $d^{-3}$ dominate. ${ }^{23,31}$ These strongly distance-dependent interactions lead to a splitting of the plasmon dipolar peak for regular one-dimensional arrays of metal nanoparticles as seen in Fig. 3(c) for ordered arrays of 50-nm Au particles. The spectral position of the extinction peak for far-field excitation shows a blueshift for polarization perpendicular to the chain axis $(T)$, and a redshift for longitudinal polarization $(L)$, which can easily be understood by analyzing Coulombic force interactions between the electrons in neighboring particles. The near-field interactions between such particles have been directly visualized using near-field optical microscopy, ${ }^{32}$ confirming a strongly enhanced field between the particles [Fig. 3(d)], indicative of near-field coupling.

One application of near-field coupling between particles in ordered arrays is the use of such structures as waveguides for electromagnetic energies at optical frequencies with a lateral mode profile below the diffraction limit of light. 6,33 Indeed, it has been shown both theoretically ${ }^{34}$ and experimentally $^{35}$ that such arrays can guide electromagnetic energy over distances of several hundred nanometers via near-field particle interactions. Such structures could potentially be used in nanoscale all-optical networks, contributing to a class of functional optical devices below the diffraction limit of light. $5,6,36$

Localized plasmon excitations mediated by particle interactions also occur in randomly nanostructured metallic surfaces. $^{37}$ In this case, multiple-scattering processes can lead to "hot spots" of extremely large field enhancement (on the order of 1000), which has enabled the use of such structures for single-molecule spectroscopy. ${ }^{38}$ 


\section{Local field enhancement around metal nanoparticle structures for sensing and nonlinear applications}

The enhanced near fields around metallic nanostructures induced by illumination at visible and near-infrared frequencies allow for a variety of intriguing applications apart from energy guiding in ordered particle arrays discussed above. Since the enhanced fields are localized to the surface of the nanostructures, they serve as a local probe of the dielectric environment within a few nanometers of the particle surface. This fact has, for example, been employed in studying variations of the local refractive index in light of biological (mass) sensing applications. ${ }^{39-41}$ Also, the local response of metallic nanostructures can serve so as to enhance the incoming and generated fields for nonlinear processes and decay rate enhancements of emissive species.

For nonlinear applications and surface-enhanced Raman sensing, the local-field $E_{\text {Local }}$ close to the metal surface should be maximized so as to maximize the respective higher-order processes, neglecting the possibility of absorption-induced damage of the optically active medium. The local-field enhancement factor $L=E_{\text {Local }} / E_{0}$, with $E_{0}$ being the amplitude of the incoming field, can for a single nanoparticle be written as the product of two factors $L$ $=L_{\mathrm{SP}}(\omega) L_{\mathrm{LR}}$, highlighting two possible enhancement processes - the surface-plasmon resonance of the whole particle $\left(L_{\mathrm{SP}}\right)$ and the lightning rod effect $\left(L_{\mathrm{LR}}\right)$. For larger particles, surface roughness and crevices can lead to additional localized resonances forming hot spots on the particle surface.

For a perfectly spherical particle in the Rayleigh limit, only the dipole surface-plasmon resonance contributes to the enhancement process, with $L_{\mathrm{SP}} \propto Q \propto T_{2}$ in the absorptiondominated regime, where $T_{2}$ and $Q$ are the dephasing time, limited by the decay of the particle plasmon into electronhole pairs and photons and by phase-destroying elastic scattering processes, and the quality factor of the resonance, respectively. The origins of plasmon decay and dephasing have been extensively discussed in the literature, ${ }^{14,42}$ and $T_{2}$ has been determined both using time-resolved pump-probe measurements ${ }^{43-46}$ and higher harmonic generation. ${ }^{47}$ For small $\mathrm{Au}$ nanospheres in air and low-index matrixes, plasmon excitation competes with interband transitions, leading to low $Q$ factors $\sim 10$, while radiation damping dominates for larger spheres with diameters of about $100 \mathrm{~nm} .{ }^{16}$ Higher $Q$ factors $\sim 20$ have been reported for spheroidal $\mathrm{Au}$ particles, due to a redshift of the long-axis dipolar resonance away from the interband transition edge. ${ }^{42}$ For Ag nanoparticles, the respective field enhancements at visible frequencies are higher, partly due to a larger spectral separation of the plasmon resonance from the interband transition edge. Another promising route to larger quality factors are metallic nanoshells, where $Q$ factors up to 150 have been estimated for Ag. ${ }^{48}$

For nonspherical shapes, the geometric and only weakly frequency-dependent lightning rod effect $L_{\mathrm{LR}}$ of the electric field at sharp surface protrusions, leading to an increased surface charge and thus a crowding of the electric-field lines, serves as an additional enhancement process. ${ }^{49-51}$ This way, highly localized fields can be generated at the tips of elongated spheroids or rough surfaces. For very rough or very high-aspect ratio particles, additionally local-plasmon resonances at specific parts on the particle surface can be excited, leading to an additional enhancement. For the case of spheroids with aspect ratios $>10: 1$, the overall particle resonance can be interpreted as an antenna effect, where the field is further enhanced at the tip due to lightning rod and local plasmon resonances. ${ }^{13,52}$ Fully analytical ${ }^{53}$ and a variety of numerical models ${ }^{54}$ have been used to quantify the field enhancement at sharp points on a variety of single-metal particles, predicting highest-field enhancement factors of about 100 for Ag particles.

The heightened optical fields near metal nanostructures manifest themselves in the enhancement of higher harmonic generation and local spectroscopy. For example, the total enhancement of second-harmonic generation on a rough silver surface is expected to scale as $L(\omega)^{4} L(2 \omega)^{2}$ upon resonance, whereas for Raman spectroscopy the enhancement scales as $L\left(\omega_{\text {exc }}\right)^{2} L\left(\omega_{\mathrm{RS}}\right)^{2}$. Note that due to the small Stokes shift in Raman scattering, usually the fields at both the excitation frequency and the Stokes frequency are enhanced. For second-harmonic generation on the other hand, usually only one of the two processes shows enhancement due to the large spectral separation between the two lines. Also, due to the significant absolute value of $|\varepsilon(\omega)|$ at visible frequencies, the field inside the particle (where second harmonic generation from the particle itself occurs) is smaller than the field outside (where SERS occurs). Thus, the observed SERS enhancements are usually significantly larger than those of second- and higher harmonic processes. Note that for calculations of field enhancement with metallic nanoparticles, the enhancement is usually evaluated for the peak power at a specific Stokes or higher harmonic output frequency, i.e., not integrated over the total resonance line shape.

Experimentally, the enhancement of second-harmonic generation at rough metal surfaces has been observed using both far-field ${ }^{55-58}$ and near-field ${ }^{59,60}$ spectroscopic techniques, with measured enhancements of second-harmonic generation on $\mathrm{Au}$ and $\mathrm{Ag}$ island films up to $1000 .{ }^{58}$ While the magnitude of the reported enhancement varies considerably, recently direct observations of localized second-harmonic enhancements of order 1000 have been reported on Au surfaces coated with random scatterers using laser scanning microscopy. ${ }^{61,62}$

The highest enhancement of an optical process on a rough metal surface so far reported is that of Raman Stokes scattering (surface-enhanced Raman scattering), where emission from single molecules ${ }^{63,64}$ with an enhancement factor of the Raman cross section up to $10^{14}$ has been observed, although the interpretation of these experiments is somewhat controversial. At this point, it is believed that this huge increase in the cross section is due to both local-field enhancements up to a factor of 1000 on roughened Ag surfaces, leading to a Raman enhancement of $10^{12}$, and to chemical effects due to adsorbate binding at the metal surface, ${ }^{65}$ making up for the additional factor of 100 in the total enhancement. Since field enhancements of a factor of 1000 can hardly be achieved for single particles, with the possible ex- 
a)

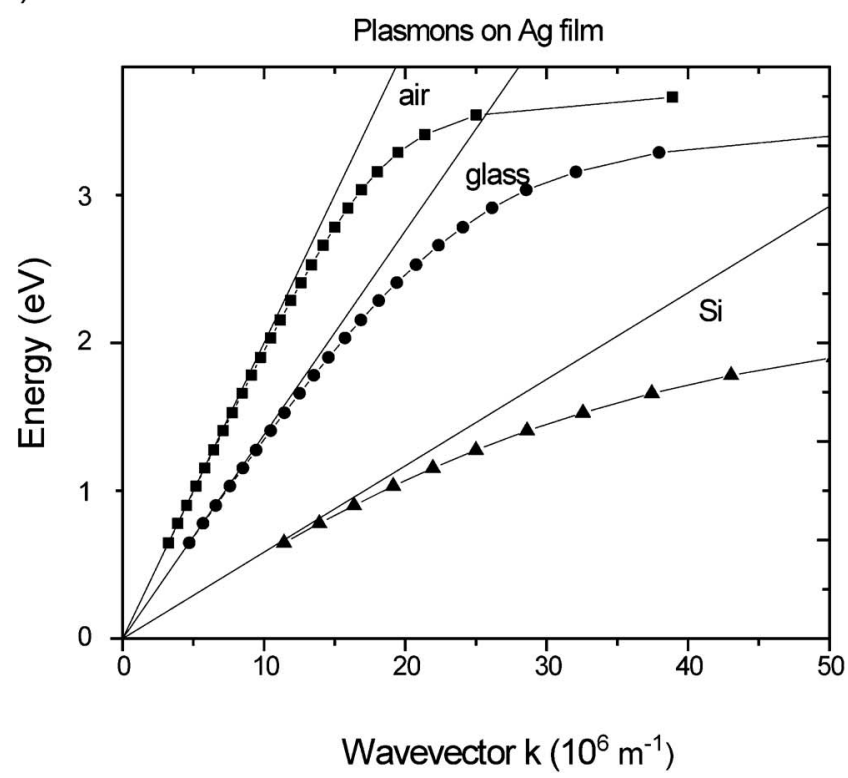

b)

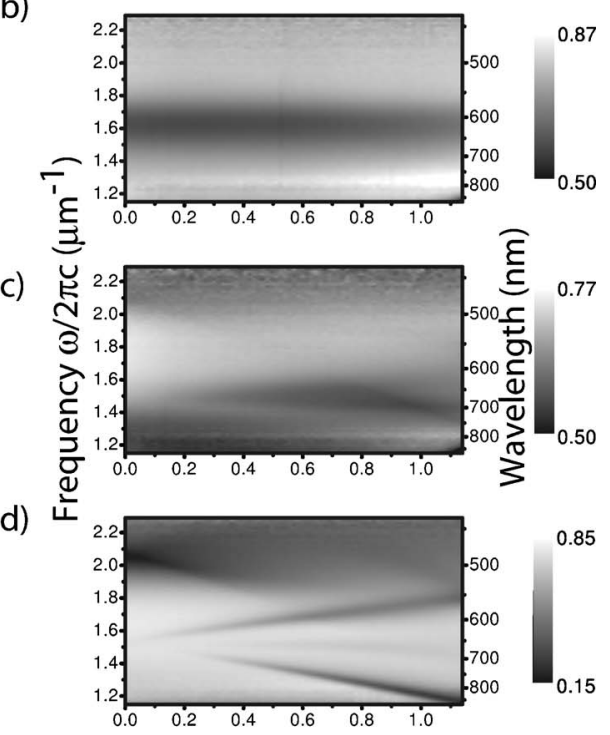

In-plane wavevector $\mathrm{k} / 2 \pi\left(\mu \mathrm{m}^{-1}\right)$

FIG. 4. (a) Calculated dispersion of surface plasmon-polaritons propagating at a $\mathrm{Ag} / \mathrm{air}, \mathrm{Ag} / \mathrm{glass}$, and $\mathrm{Ag} / \mathrm{Si}$ interface, respectively. (b)-(d) Measured transmittance as a function of in-plane wave vector and frequency for $p$-polarized light incident upon an array of nontouching nanoparticles (b), an intermediate array of bigger particles with some coalescence (c), and a periodic array of holes formed by touching nanoparticles (d), showing the transition from localized to dispersive behavior (adapted from Ref. 69).

ception of gap modes in surface crevices, it is believed that field localization in small gaps between metal particles due to geometric effects and multiple photon scattering on rough surfaces contributes to this high-field enhancement in nanometer-sized volumes, so-called hot spots, ${ }^{53,54,62}$ and recently a detailed analytical description of the enhancement using a simple resonator model has been given. ${ }^{66}$ These localized resonances tend to show very different strengths, polarization, and localization characteristics. The importance of multiple scattering for the creation of hot spots for field enhancement has been highlighted via many studies showing the significance of fractal-like character of the silver surface. $^{37,67}$

\section{INTERFACE PLASMON POLARITONS AT METAL/ DIELECTRIC BOUNDARIES}

\section{A. Surface-plasmon polaritons at metal interfaces}

Coherent electron oscillations leading to enhanced local fields at the surface of metallic structures cannot only be excited in metallic nanoparticles, but also at flat interfaces such as metallic films. As is well known, the interface between a metallic film and a dielectric can sustain SPPs in the form of coherent longitudinal charge oscillations of the conduction electrons, thus leading to a surface wave confined within one dimension perpendicular to the surface. ${ }^{3}$ At flat interfaces, these charge oscillations were observed in energy loss spectra obtained via bombardment of the film with fast electrons, revealing "low-lying plasma losses" at energies lower than the characteristic bulk-plasmon energy $\omega_{p}$ of the respective metal. ${ }^{2,68}$ At a metal/air boundary, these low-lying plasma losses for electrons occur at a frequency $\omega_{p} / \sqrt{2}$. This lowering of the plasmon resonance is due to the depolarizing effect of the flat surface, analogous to the case of localized plasmons in metallic nanoparticles. However, while for excitation with fast electrons plasma waves at flat interfaces do not propagate (group-velocity $\nu_{g}=0$ ), SPPs at lower energies exhibit a significant dispersion with wave vector $\mathbf{k}$ due to retardation effects. Figure 4(a) shows the dispersion relation for surface-plasmon polaritons propagating at a flat interface between Ag and air, glass, and silicon, respectively, calculated using a simple boundary condition analysis for electromagnetic surface waves, ${ }^{3}$ yielding

$$
k_{x}=\frac{\omega}{c}\left[\frac{\varepsilon(\omega) \varepsilon_{2}}{\varepsilon(\omega)+\varepsilon_{2}}\right),
$$

where $\varepsilon(\omega)$ are the (complex) dielectric function of the metal and $\varepsilon_{2}$ the dielectric constant of the adjacent dielectric halfspace.

As can be seen, the dispersion relations of the SPPs always lie to the right of the respective light line, approaching $\omega_{\mathrm{sp}}=\omega_{p} / \sqrt{1+\varepsilon_{2}}$ for large wave vectors, the magnitude of the wave vector at $\omega_{\text {sp }}$ being limited by dissipation. While experiments with fast electrons mainly probe this high wavevector regime where dispersion is absent, ${ }^{3}$ for lower wave vectors surface-plasmon polaritons can be excited by TMpolarized light, providing that the retardation-induced momentum mismatch is compensated. The main techniques for achieving this momentum matching are prism coupling, coupling via surface grating or roughness (defects), and using highly focused optical excitation. ${ }^{3}$ Recently, excitation of surface plasmons using regular hole arrays created via shadowed metal evaporation has been achieved. ${ }^{69}$ This work has provided a beautiful demonstration of the transition between localized surface plasmons of nontouching particles to dispersive surface-plasmon polaritons propagating along the hole film [Figs. 4(b)-4(d)]. 


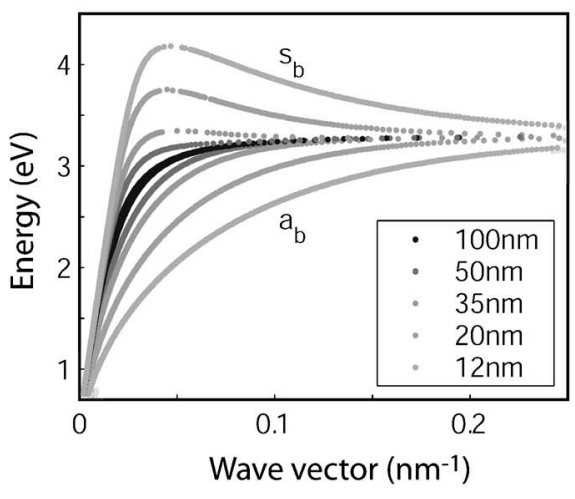

b)

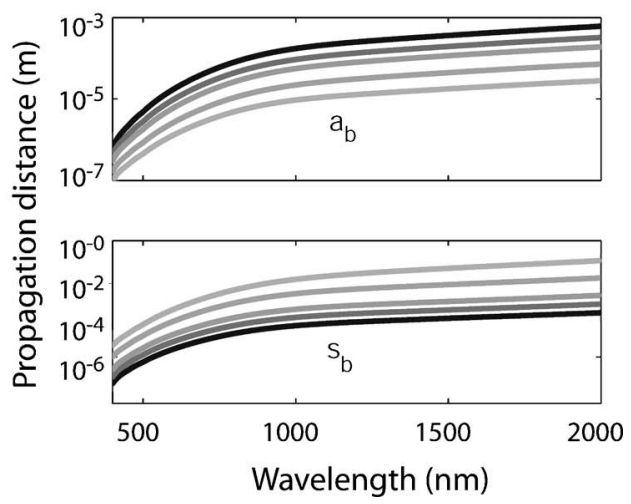

FIG. 5. (a) Dispersion relation of coupled surface plasmon modes on a thin Ag-film embedded in glass, showing the odd $\left(s_{b}\right)$ and even $\left(a_{b}\right)$ bound modes. (b) Attenuation distances for these modes (calculated using an analytical model, courtesy of Jennifer Dionne).
Due to its surface wave character, the field of a surfaceplasmon polariton decays exponentially perpendicular to the interface into the metal and dielectric. The decay length into the latter is strongly wavelength dependent, leading to high localization of the field and thus field enhancement (on the order of ten for the field amplitude at a Ag/air interface at visible frequencies) for large wave vectors. As in the case of metallic nanoparticles, this confinement and field enhancement near the metal surface have been used for applications such as evanescent surface sensors ${ }^{7}$ and for higher harmonic generation. ${ }^{58}$ Note that while the enhancement is in general more than an order of magnitude below that for metal nanoparticles, the ease of excitation of surface-plasmon polaritons using waveguide substrates has made this geometry viable for integrated optical sensors. The velocity of energy propagation, which in the low damping regime is given by the slope of the dispersion relation, and the attenuation length of the propagating plasmons, given by the imaginary part of the wave vector, both depend on the amount of field localization in the metal and dielectric near the interface, which was confirmed by directly measuring the speed of a propagating surface-plasmon polariton using pulsed excitation. ${ }^{70}$ For the visible regime, typical attenuation lengths for surfaceplasmon polaritons propagating at a $\mathrm{Ag} /$ air interface range from tens of microns to about $100 \mu \mathrm{m}$, as confirmed experimentally. For larger near-infrared wavelengths, the dielectric constants for $\mathrm{Ag}$ and $\mathrm{Au}$ in this regime ${ }^{10,71}$ predict attenuation lengths in the range of several hundred microns.

Attenuation distances on the order of a couple of tens of microns have enabled the use of patterned metallic surfaces for the creation of waveguides in a two-dimensional microoptics framework, using both local scatterers to determine the direction of energy propagation, ${ }^{72}$ and regular nanoparticle arrays on top of continuous films for the creation of surface-plasmon polariton band-gap structures and defect waveguides. $^{73}$

The exponentially decaying field inside the metal film away from the surface supporting the plasmon polariton can couple with a plasmon-polariton mode at the other surface provided that the thickness $t$ of the metal film is thin enough so that the evanescent tails of the respective modes show considerable overlap, leading to the establishment of two guided and leaky waves of opposite parities. ${ }^{74}$ For a thinmetal film embedded in a symmetric dielectric environment, the surface-plasmon frequencies of the top and bottom sur- faces are equal. Two solutions for purely bound-coupled surface-plasmon modes exist for an infinitely wide metallic thin film - a high-frequency odd mode $\left(s_{b}\right)$ showing an asymmetric distribution of the component of the electric field in the propagation direction (longitudinal component associated with the collective electron oscillations), and a lowfrequency even mode $\left(a_{b}\right)^{74,75}$ The analytically calculated dispersion and attenuation properties of these modes are shown for the case of Ag films embedded in a glass in Figs. 5(a) and 5(b), respectively. Both the $s_{b}$ and the $a_{b}$ modes exhibit marked differences with decreasing film thickness, due to the different symmetries of their spatial field distributions. The odd $s_{b}$ mode shows a node of the longitudinal electric-field component in the symmetry plane of the metal film, and penetrates less and less into the metal for decreasing thickness. In the limit of an infinitely thin film, it evolves into the fundamental TEM mode propagating in the homogenous embedding material, permitted by the symmetric distribution of the perpendicular component of the electric field. Thus, the attenuation decreases significantly with decreasing film thickness, with a concomitant decrease in confinement. This mode has thus been named a "long-ranging surfaceplasmon polariton" ${ }^{, 76}$ with multicentimeter propagation distances predicted for excitation at $\lambda>1 \mu \mathrm{m},{ }^{74}$ and has been observed experimentally shortly after its first description. ${ }^{77,78}$ Conversely, the bound even mode $\left(a_{b}\right)$ shows the opposite behavior with respect to both propagation constant and attenuation-its field becomes more and more confined to the metal with decreasing film thickness, since a mode with an asymmetric transverse electric-field component cannot exist in a homogenous background material. Both bound modes do not show a cut-off thickness for a symmetric environment.

The low attenuation of the $s_{b}$ mode for small thicknesses allows propagation distances for surface plasmons one to two orders of magnitude longer than those of a thick-metal film, ${ }^{76}$ with a concomitant increase in the magnitude of the field enhancement near the metal surface, which makes this geometry also of interest for evanescent surface sensing ${ }^{79}$ and sensing using abnormal reflection geometry permitted by the fact that the dissipative loss of long-range surfaceplasmon polaritons can be much less than their radiative losses at suitably corrugated interfaces. ${ }^{80}$

For thin metal films embedded in an asymmetric envi- 
a)
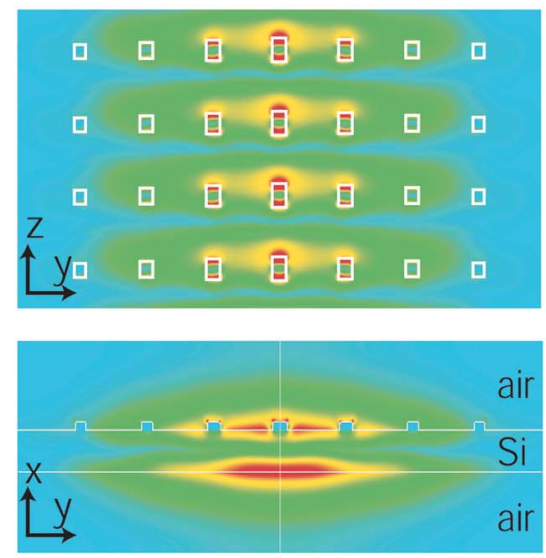

b)

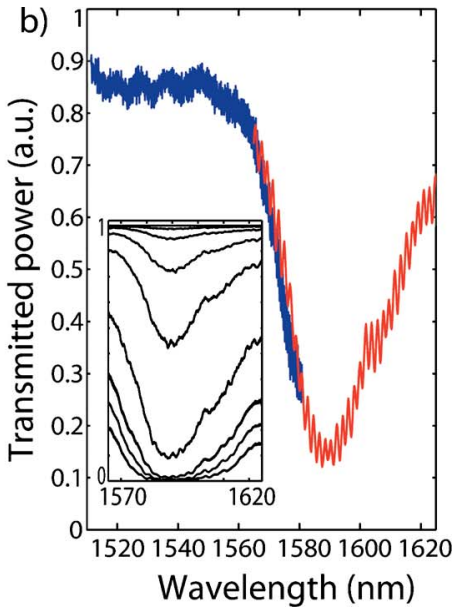

FIG. 6. (Color online) (a) Finitedifference time-domain-calculated mode profile of a fiber accessible plasmon waveguide working in highly asymmetric geometries (adapted from Ref. 88). (b) Transmission spectrum of a fiber taper placed in close proximity to the waveguide, showing resonant power transfer with efficiencies up to $75 \%$. The inset shows the evolution of the coupling with decreasing taperwaveguide gap (adapted from Ref. 89). ronment, the situation becomes more complicated due to the mismatch in surface-plasmon frequency between the lowand high-index interfaces, leading to a cut-off height for the $s_{b}$ mode, now defined as the mode of decreasing attenuation with film thickness. For only a slight dielectric mismatch between the sub- and superstrate, the possibility of long attenuation distances exists for this mode. ${ }^{81}$ However, its cutoff height increases with an increasing dielectric mismatch, lowering the propagation length. The possibility of exciting long-range plasmon modes with wave fronts tilted with respect to the propagation direction in even highly asymmetric environments has been suggested, ${ }^{82}$ or via the use of multilayer structures necessary to push the node of the longitudinal electric field into the middle of the metal film, thus yielding the lowest loss. ${ }^{80}$

\section{B. Metal stripes and nanowires: Two-dimensional confinement}

Metallic stripe waveguides with a width $w$ to thickness $t$ ratio $\gg 1$ have received considerable attention lately due to promising applications for guiding electromagnetic energy both at visible and near-infrared frequencies in the telecommunications band. In contrast with arbitrarily wide thin metal films, the modes are not purely TM in nature. As for metal films, there exists a marked difference between slabs embedded in a symmetric and asymmetric environment. ${ }^{83,84}$ In a symmetric environment, four fundamentals and a number of higher-order modes can be found and classified according to their symmetries with respect to the horizontal and vertical symmetry planes of the slabs. ${ }^{84}$ As for the infinitely wide metal film, one of the fundamental modes $\left(s s_{b}^{0}\right)$ is long ranging and of low loss for diminishing stripe thickness, and is additionally the only allowed mode below a certain cut-off thickness given by the stripe width and the dielectric environment. This mode can show low attenuation on the order of $10-0.1 \mathrm{~dB} / \mathrm{cm}$ in the telecommunications band, and has a Gaussian mode profile for sufficiently thin films, suggesting the possibility of efficient end-fire coupling techniques to conventional optical fibers, ${ }^{84}$ which has been confirmed experimentally for Au stripe waveguides excited at $1.5 \mu \mathrm{m} .^{85,86}$

For metal stripes embedded in an asymmetric environment, the situation is more complicated due to the dielectric mismatch between the sub- and superstrate. It has been shown that with decreasing slab thickness, modes can change their symmetry properties, and that all modes with decreasing attenuation for decreasing slab height show a cut-off height that increases both with decreasing width and increasing dielectric mismatch between the sub- and superstrate. ${ }^{83}$ The dielectric mismatch further allows coupling of modes of different orders between the top and bottom surfaces. Thus the modes generally show complicated spatial profiles, prohibiting efficient excitation techniques. For very large asymmetries such as glass/metal/air configurations, propagation lengths of a few microns have been experimentally demonstrated for Ag stripes of $w=1 \mu \mathrm{m}$ and $t=70 \mathrm{~nm}$ (Ref. 87) that do not support long-ranging modes in this regime.

Recently, a different approach for creating low-loss plasmon waveguides similar to stripe waveguides that show low propagation losses for even highly asymmetric environments has been described theoretically ${ }^{88}$ and demonstrated experimentally. ${ }^{89}$ The waveguides are based on a twodimensional array of metal nanoparticles, allowing additionally efficient, phase-matched evanescent excitation of such arrays using tapered fibers (Fig. 6).

The ultimate scaling down of metal stripe waveguides results in metallic nanowires with a cross section significantly smaller than the diffraction limit of light. It has recently been demonstrated that such nanowires can be used as waveguides for electromagnetic energy with a mode confinement below the diffraction limit, ${ }^{90}$ akin to the resonantly excited metal nanoparticle waveguides described above. Light transport over a few microns was observed using a far-field excitation scheme with a tapered launch-pad and near-field optical techniques for monitoring the energy transport.

\section{Apertures in a metallic screen}

SPPs are also believed to play a key role in the recently described highly wavelength-dependent enhanced transmission of light through regular arrays of subwavelength holes in an optically thick metallic screen. ${ }^{91}$ Here, it was found that not only does such a film transmit more light than can be accounted for using classical diffraction theory, ${ }^{92,93}$ but also the fraction of the transmitted light exceeds the area covered by the holes themselves. Thus, it was postulated that plasmon-assisted evanescent tunneling through the subwave- 
length apertures allows light impinging on the opaque metal surface between the holes to be transmitted via the conversion into surface plasmons. ${ }^{94}$ Intriguingly, the transmission enhancement is also present for a single aperture provided that the metallic surface on its input side shows a few periods of a regular corrugation, ${ }^{95}$ and groove cavity modes and subsequent reemission as well as aperture waveguide modes have been postulated as mechanisms for this enhancement. ${ }^{96}$ While corrugations on the input side lead to an enhanced transmission, the occurrence of highly directional beamsteering via corrugating the exit side of the screen around the aperture has been observed ${ }^{97}$ and subsequently explained in this framework. ${ }^{98}$ The wavelengths at which these effects occur can be determined via the corrugation geometry, which could make possible the creation of wavelength-selective lenses. ${ }^{99}$ However, very recently an alternate explanation for the observed effects has been presented, taking back some of the earlier claims on the amount of the transmission enhancement by presenting new experimental data. ${ }^{100}$ Theoretical and experimental studies in this area are abounding.

\section{Interactions with optically active media}

The field confinement of SPP modes at metallodielectric interfaces has been used for the enhancement of a variety of optical processes apart from those mentioned in Sec. II C, and SPPs start to be crucial components of a number of devices incorporating optically active media. Prominent recent applications include midinfrared quantum cascade lasers, where SPPs confined to the boundary between the gain medium and a thin metallic layer were found to substantially enhance the overlap between the optical mode and the gain medium and further enabled reduced cladding heights compared to semiconducting layers, ${ }^{101,102}$ and the demonstration of emission enhancement of Ref. 103 and strong coupling ${ }^{104}$ to semiconductor quantum dots.

When studying the interaction of emissive species such as quantum dots ${ }^{103}$ and wells, ${ }^{101}$ fluophores ${ }^{105}$ or rare-earth ions ${ }^{106}$ with metallic surfaces, ${ }^{107}$ gratings ${ }^{105}$ or nanoparticles, ${ }^{108}$ special care must be taken for a distinction between radiative and nonradiative contributions to the total enhanced decay rate. The huge increase of the non-radiative decay rate observed for emissive species placed in nanometric distances to metallic surfaces makes the design of plasmonic cavities for emissive devices challenging, ${ }^{109}$ but promising routes for the creation of efficient emissive devices such as light-emmiting diodes (LEDs) based on SPPsupporting cavities have been developed ${ }^{110,111}$ and partly demonstrated. ${ }^{112}$ For dipolar emitters placed into the immediate vicinity of metallic surfaces, where the dielectric continuum model, on which a macroscopic description of the metal-emitter interaction is based, breaks down, a further order-of-magnitude increase in the nonradiative decay rates based on interactions with the unscreened electrons of the metal surface has been predicted, ${ }^{113}$ which makes plasmonic devices of high promise for high-speed optical switching.

\section{OUTLOOK}

We have attempted to give the reader a limited overview of some aspects of the current state of research into the fascinating aspects and control over surface-plasmon polaritons, a subfield of nanophotonics which currently experiences an exponential growth in research efforts. Already, crucial components such as waveguides and resonators that can lead the way to a plasmonic infrastructure for highly integrated optical chips have been demonstrated and are constantly being refined. Concurrently, important advances into the control of light-matter interaction in a designed environment have been made, and it will be interesting to see how this field converges in terms of a unified description ${ }^{66}$ with the huge amount of research efforts performed on dielectric whispering-gallery-mode ${ }^{114}$ or photonic crystal cavities. ${ }^{115}$ It is anticipated that joint efforts into the further advancements of numerical design tools, fabrication as well as near-field optical characterization techniques will continue to close the size gap between optical and electronic devices.

${ }^{1}$ G. Mie, Ann. Phys. 25, 377 (1908).

${ }^{2}$ R. H. Ritchie, Phys. Rev. 106, 874 (1957).

${ }^{3}$ H. Raether, Surface Plasmons on Smooth and Rough Surfaces and on Gratings (Springer, Berlin, 1988).

${ }^{4}$ U. Kreibig and M. Vollmer, Optical Properties of Metal Clusters (Springer, Berlin, 1995).

${ }^{5}$ W. L. Barnes, A. Dereux, and T. W. Ebbesen, Nature (London) 424, 824 (2003).

${ }^{6}$ S. A. Maier, M. L. Brongersma, P. G. Kik, S. Meltzer, A. A. G. Requicha, and H. A. Atwater, Adv. Mater. (Weinheim, Ger.) 13, 1501 (2001).

${ }^{7}$ S. S. Yee, Sens. Actuators B 54, 1 (1999).

${ }^{8}$ E. Prodan, C. Radloff, N. J. Halas, and P. Nordlander, Science 302, 419 (2003).

${ }^{9}$ E. Hutter and J. H. Fendler, Adv. Mater. (Weinheim, Ger.) 16, 1685 (2004).

${ }^{10}$ P. B. Johnson and R. W. Christy, Phys. Rev. B 6, 4370 (1972).

${ }^{11}$ P. Mulvaney, MRS Bull. 26(12), 1009 (2001).

${ }^{12}$ C. F. Bohren and D. R. Huffman, Absorption and Scattering of Light by Small Particles (Wiley, New York, 1983).

${ }^{13}$ N. Calander and M. Willander, J. Appl. Phys. 92, 4878 (2002).

${ }^{14}$ S. Link and M. A. El-Sayad, Int. Rev. Phys. Chem. 19, 409 (2000).

${ }^{15}$ M. Meier and A. Wokaun, Opt. Lett. 8, 581 (1983).

${ }^{16}$ A. Wokaun, J. P. Gordon, and P. F. Liao, Phys. Rev. Lett. 48, 957 (1982).

${ }^{17}$ H. Kuwata, H. Tamaru, K. Esumi, and K. Miyano, Appl. Phys. Lett. 83(22), 4625 (2003)

${ }^{18}$ P. W. Barber and S. C. Hill, Light Scattering by Particles: Computational Methods (World Scientific, Singapore, 1990).

${ }^{19}$ W.-H. Yang, G. C. Schatz, and R. P. Van Duyne, J. Chem. Phys. 103, 869 (1995).

${ }^{20}$ K. L. Shlager and J. B. Schneider, IEEE Antennas Propag. Mag. 37, 39 (1995).

${ }^{21}$ Y. Sun and Y. Xia, Science 298, 2176 (2002).

${ }^{22}$ H. G. Craighead and G. A. Niklasson, Appl. Phys. Lett. 44, 1134 (1984).

${ }^{23}$ S. A. Maier, M. L. Brongersma, P. G. Kik, and H. A. Atwater, Phys. Rev. B 65, 193408 (2002).

${ }^{24}$ J. R. Krenn, G. Schider, W. Rechberger, B. Lamprecht, A. Leitner, and F. R. Aussenegg, Appl. Phys. Lett. 77, 3379 (2000).

${ }^{25}$ J. J. Mock, M. Barbic, D. R. Smith, D. A. Schultz, and D. Schultz, J. Chem. Phys. 116, 6755 (2002).

${ }^{26}$ T. A. Klar, M. Perner, S. Grosse, G. von Plessen, W. Spirkl, and J. Feldmann, Phys. Rev. Lett. 80, 4249 (1998).

${ }^{27}$ J. J. Penninkhof, A. Polman, L. A. Sweatlock, H. A. Atwater, A. Vredenberg, and B. J. Kooi, Appl. Phys. Lett. 83, 4137 (2003).

${ }^{28}$ R. A. McMillan, C. D. Paavola, J. Howard, S. L. Chan, N. J. Zaluzec, and J. D. Trent, Nat. Mater. 1, 247 (2002).

${ }^{29}$ B. Lamprecht, G. Schider, R. T. Lechner, H. Ditlbacher, J. R. Krenn, A. Leitner, and F. R. Aussenegg, Phys. Rev. Lett. 84, 4721 (2000).

${ }^{30}$ N. Félidj, J. Aubard, G. Lévi, J. R. Krenn, A. Hohenau, G. Schider, A. Leitner, and F. R. Aussenegg, Appl. Phys. Lett. 82, 3095 (2003). 
${ }^{31}$ S. A. Maier, P. G. Kik, and H. A. Atwater, Appl. Phys. Lett. 81, 1714 (2002).

${ }^{32}$ J. R. Krenn et al., Phys. Rev. Lett. 82, 2590 (1999).

${ }^{33}$ M. Quinten, A. Leitner, J. R. Krenn, and F. R. Aussenegg, Opt. Lett. 23, 1331 (1998)

${ }^{34}$ S. A. Maier, P. G. Kik, and H. A. Atwater, Phys. Rev. B 67, 205402 (2003).

${ }^{35}$ S. A. Maier, P. G. Kik, H. A. Atwater, S. Meltzer, E. Harel, B. E. Koel, and A. A. G. Requicha, Nat. Mater. 2, 229 (2003).

${ }^{36}$ L. Dobrzynski et al., Phys. Rev. E 69, 035601 (2004).

${ }^{37}$ V. M. Shalaev, Nonlinear Optics of Random Media (Springer, Berlin, 2000).

${ }^{38}$ K. Kneipp, H. Kneipp, I. Itzkan, R. R. Dasari, and M. S. Feld, J. Phys.: Condens. Matter 14, R597 (2002).

${ }^{39}$ J. J. Mock, D. R. Smith, and S. Schultz, Nano Lett. 3, 485 (2003).

${ }^{40}$ A. D. McFarland and R. P. Van Duyne, Nano Lett. 3, 1057 (2003).

${ }^{41}$ G. Raschke, S. Kowarik, T. Franzl, C. Sönnichsen, T. A. Klar, J. Feldmann, A. Nichtl, and K. Kürzinger, Nano Lett. 3, 935 (2003).

${ }^{42}$ C. Sönnichsen, T. Franzl, T. Wilk, G. von Plessen, J. Feldmann, O. Wilson, and P. Mulvaney, Phys. Rev. Lett. 88, 077402 (2002).

${ }^{43}$ S. L. Logunov, T. S. Ahmadi, M. A. El-Sayad, J. T. Khoury, and R. L. Whetten, J. Phys. Chem. B 101, 3713 (1997).

${ }^{44}$ T. S. Ahmadi, S. L. Logunov, and M. A. El-Sayad, J. Phys. Chem. 100, 8053 (1996).

${ }^{45}$ M. Perner et al., Phys. Rev. Lett. 78, 2192 (1997).

${ }^{46}$ S. Link, C. Burda, Z. L. Wang, and M. A. El-Sayad, J. Chem. Phys. 111, 1255 (1999).

${ }^{47}$ B. Lamprecht, J. R. Krenn, A. Leitner, and F. R. Aussenegg, Phys. Rev. Lett. 83, 4421 (1999).

${ }^{48}$ T. V. Teperik, V. V. Popov, and F. J. García de Abajo, Phys. Rev. B 69, 155402 (2004).

${ }^{49}$ J. I. Gersten, J. Chem. Phys. 72, 5779 (1980)

${ }^{50}$ J. I. Gersten and A. Nitzan, J. Chem. Phys. 73, 3023 (1980).

${ }^{51}$ P. F. Liao and A. Wokaun, J. Chem. Phys. 76, 751 (1982).

${ }^{52}$ K. B. Crozier, A. Sundaramurthy, G. S. Kino, and C. F. Quate, J. Appl. Phys. 94, 4632 (2003).

${ }^{53}$ H. Xu, J. Aizpurua, M. Käll, and P. Apell, Phys. Rev. E 62, 4318 (2000).

${ }^{54}$ E. Hao and G. C. Schatz, J. Chem. Phys. 120, 357 (2004).

${ }^{55}$ C. K. Chen, T. F. Heinz, D. Ricard, and Y. R. Shen, Phys. Rev. B 27, 1965 (1983).

${ }^{56}$ G. T. Boyd, T. Rasing, J. R. R. Leite, and Y. R. Shen, Phys. Rev. B 30, 519 (1984)

${ }^{57}$ A. Podlipensky, J. Lange, G. Seifert, H. Graener, and I. Cravetchi, Opt. Lett. 28, 716 (2003).

${ }^{58}$ A. Wokaun, J. G. Bergman, J. P. Heritage, A. M. Glass, P. F. Liao, and D. H. Olson, Phys. Rev. B 24, 849 (1981).

${ }^{59}$ I. I. Smolyaninov, A. V. Zayats, and C. C. Davis, Phys. Rev. B 56, 9290 (1997).

${ }^{60}$ A. V. Zayats, T. Kalkbrenner, V. Sandoghdar, and J. Mlynek, Phys. Rev. B 61, 4545 (2000).

${ }^{61}$ J. Beermann and S. I. Bozhevolnyi, Phys. Rev. B 69, 155429 (2004).

${ }^{62}$ S. I. Bozhevolnyi, J. Beermann, and V. Coello, Phys. Rev. Lett. 90, 197403 (2003).

${ }^{63}$ K. Kneipp, Y. Wang, H. Kneipp, L. T. Perelman, I. Itzkan, R. Dasari, and M. S. Feld, Phys. Rev. Lett. 78, 1667 (1997).

${ }^{64}$ S. M. Nie and S. R. Emery, Science 275, 1102 (1997).

${ }^{65}$ J. I. Gersten and A. Nitzan, J. Chem. Phys. 75, 1139 (1981).

${ }^{66}$ S. A. Maier and O. Painter (unpublished).

${ }^{67}$ Z. Wang, S. Pan, T. D. Krauss, H. Du, and L. J. Rothberg, Proc. Natl. Acad. Sci. U.S.A. 100, 8638 (2003).

${ }^{68}$ C. J. Powell and J. B. Swan, Phys. Rev. 118, 640 (1960).

${ }^{69}$ W. A. Murray, S. Astilean, and W. L. Barnes, Phys. Rev. B 69, 165407 (2004).

${ }^{70}$ M. Bai, C. Guerrero, S. Ioanid, E. Paz, M. Sanz, and N. García, Phys. Rev. B 69, 115416 (2004).

${ }^{71}$ E. D. Palik, Handbook of Optical Constants (Academic, London, 1985).

${ }^{72}$ H. Ditlbacher, J. R. Krenn, G. Schider, A. Leitner, and F. R. Aussenegg, Appl. Phys. Lett. 81, 1762 (2002).
${ }^{73}$ S. I. Bozhevolnyi, J. Erland, K. Leosson, P. M. W. Skovgaard, and J. M. Hvam, Phys. Rev. Lett. 86, 3008 (2001).

${ }^{74}$ J. J. Burke, G. I. Stegeman, and T. Tamir, Phys. Rev. B 33, 5186 (1986).

${ }^{75}$ E. N. Economou, Phys. Rev. 182, 539 (1969).

${ }^{76}$ D. Sarid, Phys. Rev. Lett. 47, 1927 (1981).

${ }^{77}$ A. E. Craig, G. A. Olson, and D. Sarid, Opt. Lett. 8, 380 (1983).

${ }^{78}$ J. C. Quail, J. G. Rako, and H. J. Simon, Opt. Lett. 8, 377 (1983).

${ }^{79}$ D. Sarid, R. T. Deck, A. E. Craig, R. K. Hickernell, R. S. Jameson, and J. J. Fasano, Appl. Opt. 21, 3993 (1982).

${ }^{80}$ F. Pigeon, I. F. Salakhutdinov, and A. V. Tishchenko, J. Appl. Phys. 90, 852 (2001).

${ }^{81}$ B. Prade, J. Y. Vinet, and A. Mysyrowicz, Phys. Rev. B 44, 13556 (1991).

${ }^{82}$ M. N. Zervas, Opt. Lett. 16, 720 (1991).

${ }^{83}$ P. Berini, Phys. Rev. B 63, 125417 (2001).

${ }^{84}$ P. Berini, Phys. Rev. B 61, 10484 (2000).

${ }^{85}$ R. Charbonneau, P. Berini, E. Berolo, and E. Lisicka-Shrzek, Opt. Lett. 25, 844 (2000).

${ }^{86}$ T. Nikolajsen, K. Leosson, I. Salakhutdinov, and S. I. Bozhevolnyi, Appl. Phys. Lett. 82, 668 (2003).

${ }^{87}$ B. Lamprecht et al., Appl. Phys. Lett. 79, 51 (2001).

${ }^{88}$ S. A. Maier, P. E. Barclay, T. J. Johnson, M. D. Friedman, and O. Painter, Appl. Phys. Lett. 84, 3990 (2004).

${ }^{89}$ S. A. Maier, M. D. Friedman, P. E. Barclay, and O. Painter, Appl. Phys. Lett. 86, 071103 (2005)

${ }^{90}$ J. R. Krenn, B. Lamprecht, H. Ditlbacher, G. Schider, M. Salerno, A. Leitner, and F. R. Aussenegg, Europhys. Lett. 60, 663 (2002).

${ }^{91}$ T. W. Ebbesen, H. J. Lezec, H. F. Ghaemi, T. Thio, and P. A. Wolff, Nature (London) 391, 667 (1998).

${ }^{92}$ H. A. Bethe, Phys. Rev. 66, 163 (1944).

${ }^{93}$ C. J. Bouwkamp, Philips Res. Rep. 5, 321 (1950).

${ }^{94}$ L. Martín-Moreno, F. J. García-Vidal, H. J. Lezec, K. M. Pellerin, T. Thio, J. B. Pendry, and T. W. Ebbesen, Phys. Rev. Lett. 86, 1114 (2001).

${ }^{95}$ D. E. Grupp, H. J. Lezec, T. Thio, and T. W. Ebbesen, Adv. Mater. (Weinheim, Ger.) 11, 860 (1999).

${ }^{96}$ F. J. García-Vidal, H. J. Lezec, T. W. Ebbesen, and L. Martin-Moreno, Phys. Rev. Lett. 90, 213901 (2003).

${ }^{97}$ H. J. Lezec, A. Degiron, E. Devaux, R. A. Linke, L. Martin-Moreno, F. J. Garcia-Vidal, and T. W. Ebbesen, Science 297, 820 (2002).

${ }^{98}$ L. Martín-Moreno, F. J. García-Vidal, H. J. Lezec, A. Degiron, and T. W. Ebbesen, Phys. Rev. Lett. 90, 167401 (2003).

${ }^{99}$ F. J. García-Vidal, L. Martín-Moreno, H. J. Lezec, and T. W. Ebbesen, Appl. Phys. Lett. 83, 4500 (2003).

${ }^{100}$ H. J. Lezec and T. Thio, Opt. Express 12, 3629 (2004).

${ }^{101}$ C. Sirtori, C. Gmachl, F. Capasso, J. Faist, D. L. Sivco, A. L. Hutchison, and A. Y. Cho, Opt. Lett. 23, 1366 (1998).

${ }^{102}$ A. Tredicucci, C. Gmachl, F. Capasso, J. E. Hutchison, D. L. Sivco, and A. Y. Cho, Appl. Phys. Lett. 76, 2164 (2000).

${ }^{103}$ K. T. Shimizu, W. K. Woo, B. R. Fisher, H. J. Eisler, and M. G. Bawendi, Phys. Rev. Lett. 89, 117401 (2002).

${ }^{104}$ J. Bellessa, C. Bonnand, J. C. Plenet, and J. Mugnier, Phys. Rev. Lett. 93, 036404 (2004).

${ }^{105}$ P. Andrew and W. L. Barnes, Phys. Rev. B 64, 125405 (2001).

${ }^{106}$ R. M. Amos and W. L. Barnes, Phys. Rev. B 55, 7249 (1997).

${ }^{107}$ E. A. Hinds, in Cavity Quantum Electrodynamics, edited by P. Berman, (Academic, Boston, 1994), p. 1.

${ }^{108}$ E. Dulkeith et al., Phys. Rev. Lett. 89, 203002 (2002).

${ }^{109}$ J. A. E. Wasey and W. L. Barnes, J. Mod. Opt. 47, 725 (2000).

${ }^{110}$ S. C. Kitson, W. L. Barnes, and J. R. Sambles, J. Appl. Phys. 84, 2399 (1998).

${ }^{111}$ W. L. Barnes, J. Lightwave Technol. 17, 2170 (1999).

${ }^{112} \mathrm{~J}$. Vuckovic, M. Loncar, and A. Scherer, IEEE J. Quantum Electron. 36(10), 1131 (2000).

${ }^{113}$ I. A. Larkin, M. I. Stockman, M. Achermann, and V. I. Klimov, Phys. Rev. B 69, 012403 (2004).

${ }^{114}$ R. K. Chang and A. L. Campillo, Optical Processes in Microcavities (World Scientific, Singapore, 1996).

115 J. D. Joannopoulos, R. D. Meade, and J. N. Winn, Photonic CrystalsMolding the Flow of Light. (Princeton University Press, Princeton, 1995). 